\title{
Toward an Alternative Intrinsic Probe for Spectroscopic Characterization of a Protein
}

\author{
Nirmal Goswami, Abhinandan Makhal, and Samir Kumar Pal* \\ Department of Chemical, Biological and Macromolecular Sciences, Unit for Nano Science and Technology, S. \\ N. Bose National Centre for Basic Sciences, Block JD, Sector III, Salt Lake, Kolkata 700 098, India
}

Received: June 28, 2010; Revised Manuscript Received: September 27, 2010

\begin{abstract}
The intrinsic fluorescent amino acid tryptophan is the unanimous choice for the spectroscopic investigation of proteins. However, several complicacies in the interpretation of tryptophan fluorescence in a protein are inevitable and an alternative intrinsic protein probe is a longstanding demand. In this contribution, we report an electron-transfer reaction in a human transporter protein (HSA) cavity which causes the tryptophan residue (Trp214) to undergo chemical modification to form one of its metabolites kynurenine (Kyn214). Structural integrity upon modification of the native protein is confirmed by dynamic light scattering (DLS) as well as near and far circular dichroism (CD) spectroscopy. Femtosecond-resolved fluorescence transients of the modified protein describe the dynamics of solvent molecules in the protein cavity in both the native and denatured states. In order to establish general use of the probe, we have studied the dipolar interaction of Kyn214 with a surface-bound ligand (crystal violet, CV) of the protein. By using the sensitivity of FRET, we have determined the distance between Kyn214 (donor) and CV (acceptor). Our study is an attempt to explore an alternative intrinsic fluorescence probe for the spectroscopic investigation of a protein. In order to establish the efficacy of the modification technique we have converted the tryptophan residues of other proteins (bovine serum albumin, chymotrypsin and subtilisin Carlsberg) to kynurenine and confirmed their structural integrity. We have also shown that catalytic activity of the enzymes remains intact upon the modification.
\end{abstract}

\section{Introduction}

Proteins represent essential building blocks behind cell structure and the engines supporting every metabolic reaction. Fluorescence spectroscopy is a powerful technique for studying various aspects of protein biophysics, such as protein folding, protein-protein interactions, and protein dynamics. ${ }^{1,2}$ Tagging a protein with extrinsic fluorophore is an important and valuable tool for studying structure and microenvironment. Extrinsic fluorescent probes, such as dansyl or related amino acid derivatives, can also be incorporated into proteins via modification of reactive residues, such as lysine and cysteine, or via chemical synthesis. ${ }^{3,4}$ In addition, quantum dots are also useful to know the energy transfer efficiency in protein. ${ }^{5}$ However, one of the major disadvantages is the possibility of perturbation of structural integrity of the protein upon incorporation of the extrinsic probe. Moreover, the bulky nature of the external probe is not suitable for the spectroscopic characterization of a protein such as protein folding, energy transfer, etc. An alternative choice for the spectroscopic studies of the proteins is intrinsic fluoroprobes. Most proteins contain amino acid residues that are intrinsically fluorescent: tryptophan, tyrosine, and phenylalanine. Among those, tryptophan is the most attractive fluorophore in protein. ${ }^{6}$ However, critical wavelength of excitation $(295 \mathrm{~nm}-300 \mathrm{~nm})$ in order to avoid interference of the other fluorescent amino acids (tyrosine and phenyl alanine) and the complicacy in data interpretation due to ultrafast internal conversion $^{7,8}$ make spectroscopic studies involving tryptophan as probe to be a bit difficult. Relatively inexpensive laser sources

* Corresponding author. E-mail: skpal@bose.res.in. Presently: Arthur Amos Noyes Laboratory of Chemical Physics, California Institute of Technology, 1200 East California Boulevard, Pasadena, CA 91125, U.S.A. Email: spal@caltech.edu. in the desired excitation wavelength $(295 \mathrm{~nm})$ are also a problem for such investigations.

Exploration of an intrinsic probe which could be alternative to tryptophan with an excitation wavelength at near-visible wavelength $(\sim 375 \mathrm{~nm})$ and eligible for the spectroscopic studies of a protein within structural integrity is the motive of our present work. It has been reported that the oxidation of tryptophan in bulk solution results in the formation of a number of byproducts, such as ditryptophan, kynurenine, 3-hydroxykynurenine, $N$-formylkynurenine, and some cross-linked products, formed by reaction with these byproducts. ${ }^{9-15}$ Each of these byproducts has a specific absorption and shows strong emission, which provides the basis for identifying a particular species. However, oxidation of tryptophan in protein environment is not well studied. In this regard, we have modified Trp214 of a model single tryptophan protein human serum albumin (HSA) to kynurenine (Kyn214) by electron-transfer reaction. Kynurenine is one of the tryptophan metabolites and omnipresent in the lens of human eyes in order to protect retina from possible UV $(300-400 \mathrm{~nm})$ radiation damage. ${ }^{16}$ Structural integrity of the protein upon the modification is confirmed by circular dichroism (CD) spectroscopy. Similar unfolding pathways are confirmed by dynamic light scattering (DLS) and near- and far-UV CD. In order to compare the efficacy of the Kyn214 for the spectroscopic studies of the protein, we have also excited Trp214 of the native protein. Here we have demonstrated that Kyn214 is equally capable compared to Trp214 in order to unravel various folded states of the protein. The femtosecond-resolved dynamics of hydration as revealed by Trp214 in the domain II can also be monitored by Kyn214. Finally, we have shown that the probe can be used for the dipolar interaction with other acceptor protein-bound dyes in order to study FRET. ${ }^{17,18}$ Our modification technique has also been applied to other proteins including enzymes within their structural integrity. For the 
enzymes we have confirmed that modification of tryptophan to kynurenine has minimal effect on their catalytic activity.

\section{Materials and Methods}

Human serum albumin (HSA), bovine serum albumin (BSA), subtilisin Carlsberg (SC), $\alpha$-chymotrypsin (CHT), L-kynurenine, $\mathrm{CV}$, acetonitrile (ACN), Ala-Ala-Phe 7-amido-4-methyl coumarin (AAF-AMC), N-CBZ-Gly-Gly-Leu $p$-nitroanilide (CBZGGL-pNA), and phosphate buffer were obtained from Sigma. The electron-accepting $p$-benzoquinone (BQ) was from Alfa Aesar. Double-distilled water was used for preparation of aqueous solutions. Aqueous-organic solution (40\% ACN, v/v) was prepared in a phosphate buffer (final concentration is 20 $\mathrm{mM}$ ) at $\mathrm{pH}$ 7.0. A stock solution of HSA was prepared in 20 $\mathrm{mM}$ phosphate buffer solution. Ethanol and sodium hydroxide were obtained from Merck. Modified HSA solution was prepared by the addition of $14 \mu \mathrm{M}$ BQ solution, and we have estimated the yield of kynurenine bound protein and found to be $\sim 70 \%$. HSA was labeled with $\mathrm{CV}$ as follows: about $1 \mathrm{mg}$ of $\mathrm{CV}$ was dissolved in $2 \mathrm{~mL}$ of double-distilled water and from there $20 \mu \mathrm{L}$ of concentrate $\mathrm{CV}$ solution was injected to $2 \mathrm{~mL}$ of phosphate buffer containing $20 \mu \mathrm{M}$ modified HSA. In order to ensure better energy transfer, the mixture was allowed to stir for $1 \mathrm{~h}$. Sodium hydroxide solution was used for chemical denaturation. Catalytic activity measurements of CHT and SC were made using the chromogenic synthetic substrates AAFAMC and CBZ-GGL-pNA as per the conventional procedure. Concentrations of the substrates were determined using $\epsilon_{325}=$ $16 \mathrm{mM}^{-1} \mathrm{~cm}^{-1}$ for AAF-AMC in buffer $\mathrm{pH}=7.0$, and $\epsilon_{315}=$ $14 \mathrm{mM}^{-1} \mathrm{~cm}^{-1}$ for CBZ-GGL-pNA in water. The extinction coefficients of the products formed are $7.6 \mathrm{mM}^{-1} \mathrm{~cm}^{-1}$ (for $\mathrm{AMC}$ at $370 \mathrm{~nm}$ ) and $8.8 \mathrm{mM}^{-1} \mathrm{~cm}^{-1}$ (for pNA at $410 \mathrm{~nm}$ ). For the enzymatic kinetics experiment, the enzyme concentration was maintained at $1 \mu \mathrm{M}$ while that of substrate was maintained at 35 and $185 \mu \mathrm{M}$ for AMC and pNA respectively. The rate of formation of product was monitored using the change in absorbance of the product with time.

Steady-state absorption and emission were measured with a Shimadzu UV-2450 spectrophotometer and Jobin Yvon Fluoromax-3 fluorimeter, respectively. DLS measurements were done with Nano $\mathrm{S}$ Malvern instrument employing a $4 \mathrm{~mW}$ $\mathrm{He}-\mathrm{Ne}$ laser $(\lambda=632.8 \mathrm{~nm})$ equipped with a thermostated sample chamber. All the scattered photons were collected at $173^{\circ}$ scattering angle. The scattering intensity data were processed using the instrumental software to obtain the hydrodynamic diameter $\left(d_{\mathrm{H}}\right)$ and the size distribution of the scatterer in each sample. The instrument measures the time-dependent fluctuation in the intensity of light scattered from the particles in solution at a fixed scattering angle. Hydrodynamic diameter $\left(d_{\mathrm{H}}\right)$ of the proteins is estimated from the intensity autocorrelation function of the time-dependent fluctuation in intensity. $d_{\mathrm{H}}$ is defined as

$$
d_{\mathrm{H}}=\frac{k_{\mathrm{B}} T}{3 \pi \eta D}
$$

where $k_{\mathrm{B}}$ is the Boltzmann constant, $\eta$ is the viscosity, and $D$ is the translational diffusion coefficient. In a typical size distribution graph from the DLS measurement, the $X$-axis shows a distribution of size classes in nanometers, while the $Y$-axis shows the relative intensity of the scattered light. Fluorescence decay transients were measured and fitted by using a commercially available spectrophotometer (LifeSpec-ps, Edinburgh Instru- ments, UK) with an $60 \mathrm{ps}$ instrument response function (IRF) and an attachment for temperature-dependent studies (Julabo, Model F32). The tryptophan residues of the proteins were excited by using the third harmonic laser beam of the $900 \mathrm{~nm}$ from a mode-locked Ti-sapphire laser with $80 \mathrm{MHz}$ repetition rate (Tsunami, Spectra Physics), pumped by a $10 \mathrm{~W}$ Millennia (Spectra Physics) followed by a pulse peaker (rate $8 \mathrm{MHz}$ ). The CD spectrum was measured in a Jasco 815 spectropolarimeter with a Peltier setup for the temperature-dependent measurements. CD studies were done with $10 \mathrm{~mm}$ path length cell. Thermal unfolding of HSA and modified HSA was monitored by recording far-UV CD at $222 \mathrm{~nm}$ and near-UV CD at 268 $\mathrm{nm}$ as a function of temperature. The temperature was raised from 20 to $90{ }^{\circ} \mathrm{C}$ in $2{ }^{\circ} \mathrm{C}$ step with 1 or 2 min equilibration time at each temperature. The unfolding curves were constructed after converting the $\mathrm{CD}$ values to fraction unfolded.

The temperature-dependent femtosecond-resolved fluorescence is measured using a femtosecond upconversion setup (FOG 100, CDP) along with an indigenous temperature controller setup. The sample is excited at $375 \mathrm{~nm}$, using the second harmonic of a mode-locked $\mathrm{Ti}-$ sapphire laser with an $80 \mathrm{MHz}$ repetition rate (Tsunami, Spectra Physics), pumped by $10 \mathrm{~W}$ Millennia (Spectra Physics). The fundamental beam is frequency doubled in a nonlinear crystal $\left(1 \mathrm{~mm} \mathrm{BBO}, q=25^{\circ}, f=90^{\circ}\right)$. The fluorescence emitted from the sample is up-converted in a nonlinear crystal $\left(0.5 \mathrm{~mm} \mathrm{BBO}, q=10^{\circ}, f=90^{\circ}\right)$ using a gate pulse of the fundamental beam. The upconverted light is dispersed in a double monochromator and detected using photon counting electronics. A cross-correlation function obtained using the Raman scattering from water displayed a full width at halfmaximum (fwhm) of 165 fs. The femtosecond fluorescence decays were fitted using SCIENTIST software.

To estimate the FRET efficiency of the donor and hence to determine the distance of the donor-acceptor pair, we followed the methodology described in chapter 13 of ref 1 . The Förster distance $\left(R_{0}\right)$ is given by

$$
R_{0}=0.211\left[\kappa^{2} n^{-4} Q_{\mathrm{D}} J(\lambda)\right]^{1 / 6} \quad(\text { in } \AA)
$$

where $\kappa^{2}$ is a factor describing the relative orientation in space of the transition dipoles of the donor and acceptor. In the present study, the acceptor CV can bind at all the surface of the protein HSA at any orientation with respect to the donor Kyn. Thus, for an ensemble of donor-acceptor $(\mathrm{D}-\mathrm{A})$ pairs the relative orientation of the $\mathrm{D}-\mathrm{A}$ is not supposed to be constant. We

\section{SCHEME 1: Modification of Trp214 of a Model} Globular Protein (HSA) as a Result of Electron-Transfer Reaction with $p$-Benzoquinone (BQ) in the Protein Cavity (left); Product of the Electron Transfer Reaction Kynurenine (Kyn214) in the Protein Cavity (right) ${ }^{a}$
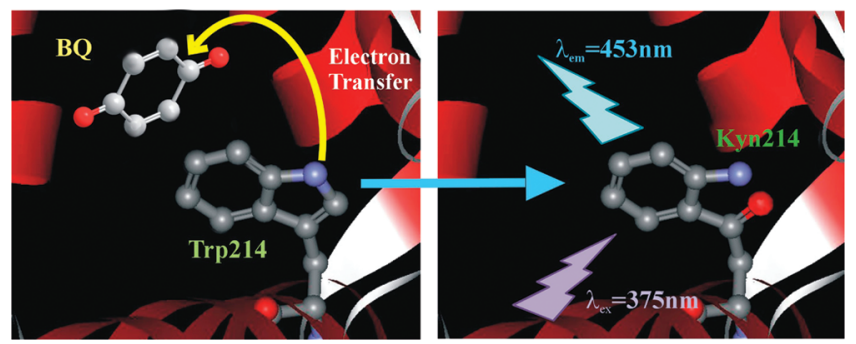

${ }^{a}$ A typical excitation wavelength $(375 \mathrm{~nm})$ used in our experiment and emission peak of the modified probe Kyn214 at $453 \mathrm{~nm}$ are also shown in the schematic. 

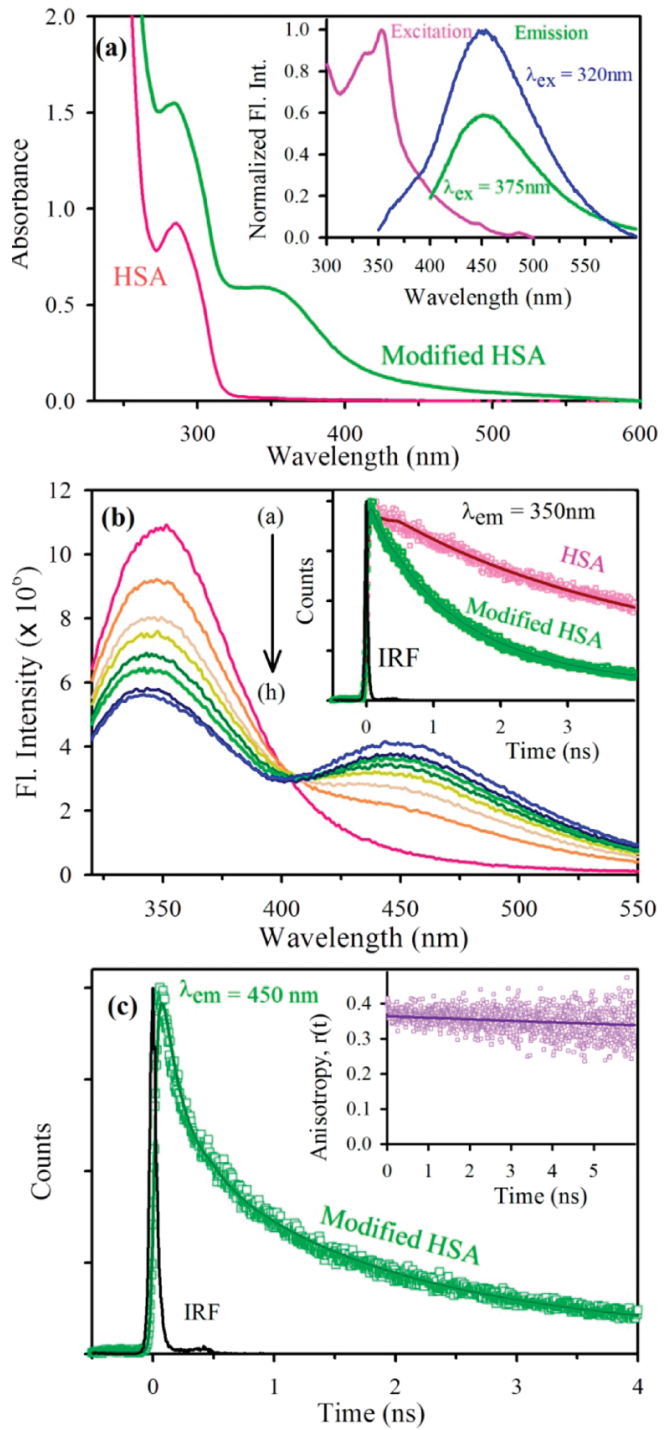

Figure 1. (a) Absorption spectrum of HSA and modified HSA. The spectra are recorded at room temperature with a concentration of 20 $\mu \mathrm{M}$ for both modified and nonmodified HSA in sodium phosphate buffer ( $\mathrm{pH} 7.0,20 \mathrm{mM}$ ). Inset shows the excitation and emission spectra of modified HSA. (b) Steady-state fluorescence spectrum of HSA in phosphate buffer solution using different BQ concentrations $(\mathrm{a}-\mathrm{h} ; 0-14$ $\mu \mathrm{M})$. Inset shows the picosecond-resolved fluorescence transients of protein before and after modification (excitation wavelength $=299 \mathrm{~nm}$ ). (c) Picosecond-resolved fluorescence transients of modified HSA collected at $450 \mathrm{~nm}$ (excitation wavelength $=375 \mathrm{~nm}$ ). The fluorescence anisotropy of Kyn 214 residue in $\mathrm{HSA}$ at $20^{\circ} \mathrm{C}$ is presented in the inset.

assumed that the orientation factor $\kappa^{2}$ is equal to the dynamic average of $2 / 3$ which is not a major deviation from real fact of randomized donor and acceptor orientations in an ensemble. Moreover, variation of $\kappa^{2}$ does not seem to have resulted in major errors in the calculated distances. ${ }^{19}$ The refractive index (n) of the medium was assumed to be 1.4. $J(\lambda)$, the overlap integral, which expresses the degree of spectral overlap between the donor emission and the acceptor absorption is given by

$$
J(\lambda)=\frac{\int_{0}^{\infty} F_{\mathrm{D}}(\lambda) \varepsilon(\lambda) \lambda^{4} \mathrm{~d} \lambda}{\int_{0}^{\infty} F_{\mathrm{D}}(\lambda) \mathrm{d} \lambda}
$$

where $F_{\mathrm{D}}(\lambda)$ is the fluorescence intensity of the donor in the wavelength range of $\lambda$ to $\lambda+\mathrm{d} \lambda$ and is dimensionless. $\varepsilon(\lambda)$ is
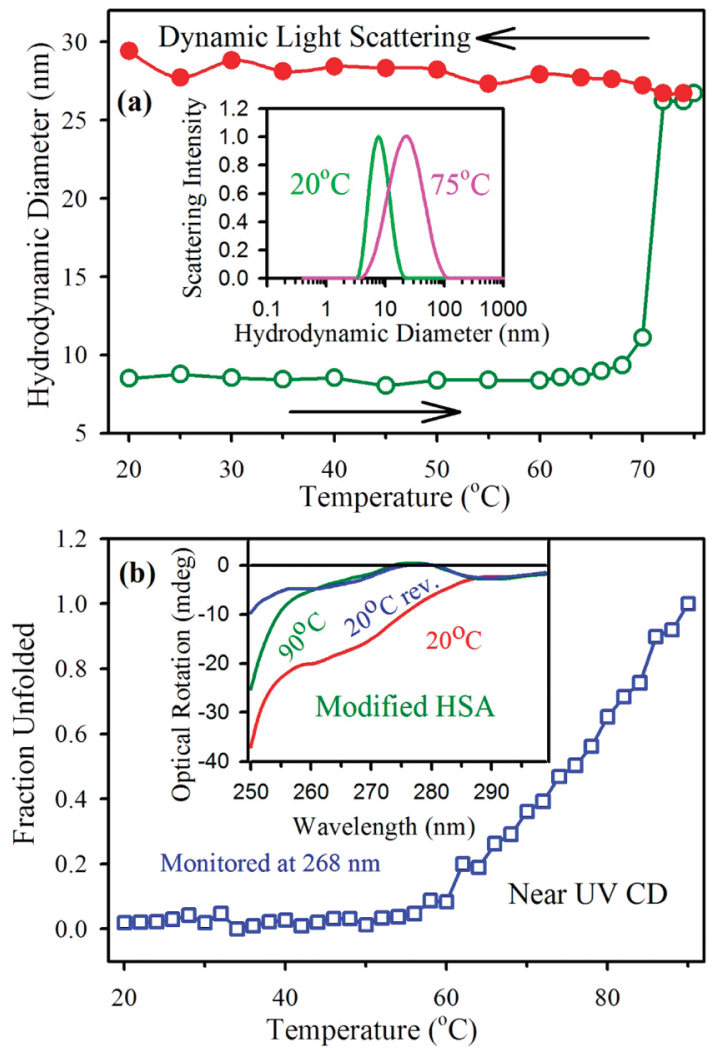

Figure 2. (a) Effect of temperature on the size of modified HSA in $20 \mathrm{mM}$ phosphate buffer. Open symbols represent forward (unfolding) process, and closed symbols represent backward (refolding) process. DLS signals of modified HSA at 25 and $75{ }^{\circ} \mathrm{C}$ are presented in the inset. (b) Thermal denaturation plots at $268 \mathrm{~nm}$ obtained by near-UV $\mathrm{CD}$, from 20 to $90{ }^{\circ} \mathrm{C}$. Inset shows the temperature dependence of the near-UV CD spectrum.

the extinction coefficient (in $\mathrm{M}^{-1} \mathrm{~cm}^{-1}$ ) of the acceptor at $\lambda$. If $\lambda$ is in nanometers, then $J$ is in units of $\mathrm{M}^{-1} \mathrm{~cm}^{-1} \mathrm{~nm}^{4}$. The estimated value of the overlap integral is $5.5146 \times 10^{15} \mathrm{M}^{-1}$ $\mathrm{cm}^{-1} \mathrm{~nm}^{4}$.

$Q_{\text {D }}$, the quantum yield of the donor in the absence of acceptor, was calculated according to the equation ${ }^{20}$

$$
Q=Q_{\mathrm{R}}\left(\frac{I}{I_{\mathrm{R}}}\right)\left(\frac{\mathrm{OD}_{R}}{\mathrm{OD}}\right)\left(\frac{n^{2}}{n_{\mathrm{R}}^{2}}\right)
$$

where $Q$ and $Q_{\mathrm{R}}$ are the quantum yield of the protein and reference (L-kynurenine in water), $I$ and $I_{\mathrm{R}}$ are the integrated fluorescence intensities of the protein and reference, OD and $\mathrm{OD}_{\mathrm{R}}$ are the optical densities of the protein and reference at the excitation wavelength, and $n$ and $n_{\mathrm{R}}$ are the refractive indices of the protein and reference solutions. The absolute quantum yield of kynurenine ${ }^{16}$ in water was taken to be $8.2 \times 10^{-4}$. Refractive indices of the solutions are measured by using Rudolph J357 automatic refractometer. The quantum yield of kynurenine in HSA was found to be $48 \times 10^{-4}$.

Once the value of $R_{0}$ is known, the donor-acceptor distance $(R)$ can easily be calculated using the formula

$$
R^{6}=\left[R_{0}{ }^{6}(1-E)\right] / E
$$

Here $E$ is FRET efficiency, measured by using the lifetimes of the donor in the absence $\left(\tau_{\mathrm{D}}\right)$ and presence $\tau_{\mathrm{DA}}$ of acceptor which is defined as 

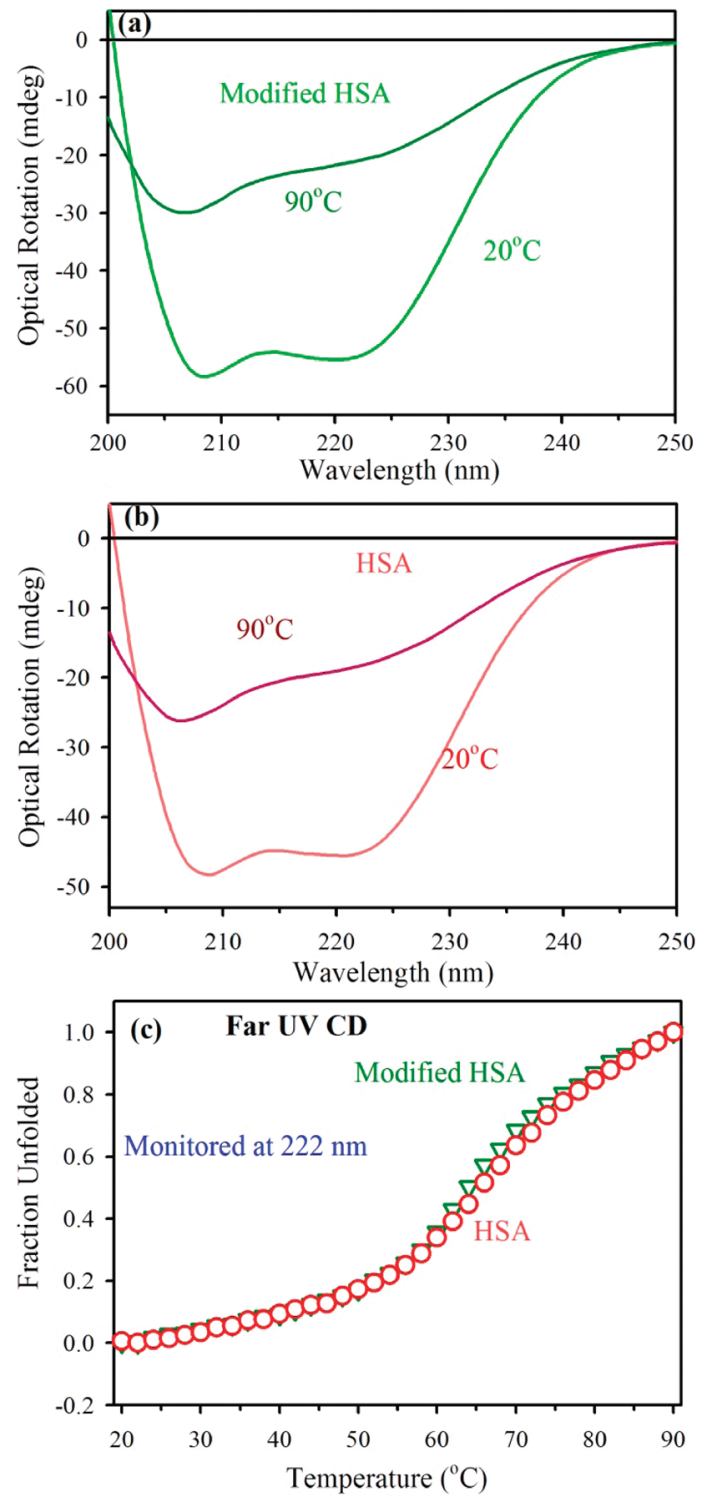

Figure 3. Far-UV CD spectrum of (a) modified HSA in sodium phosphate buffer ( $\mathrm{pH} 7.0,20 \mathrm{mM}$ ). (b) HSA in sodium phosphate buffer $(\mathrm{pH} 7.0,20 \mathrm{mM})$. In both cases, the spectra are recorded at 20 and 90 ${ }^{\circ} \mathrm{C}$. (c) Thermal denaturation plots at $222 \mathrm{~nm}$ obtained by far-UV CD, from 20 to $90{ }^{\circ} \mathrm{C}$. Transition temperature $\left(T_{\mathrm{m}}\right.$, the midpoint of unfolding transition) is $70{ }^{\circ} \mathrm{C}$ for both (HSA and modified HSA) in phosphate buffer.

$$
E=1-\tau_{\mathrm{DA}} / \tau_{\mathrm{D}}
$$

It has to be noted that eq 6 holds rigorously only for a homogeneous system (i.e., identical donor-acceptor complexes) in which the donor and the donor-acceptor complex have single-exponential decays. However, for donor-acceptor systems decaying with multiexponential lifetimes, ${ }^{1}$ FRET efficiency $(E)$ is calculated from the amplitude-weighted lifetimes $\langle\tau\rangle=$ $\sum_{i} \alpha_{i} \tau_{i}$ where $\alpha_{\mathrm{i}}$ is the relative amplitude contribution to the lifetime $\tau_{i}$. We have used the amplitude-weighted time constants for $\tau_{\mathrm{D}}$ and $\tau_{\mathrm{DA}}$ to evaluate $E$ using eq 6 .

\section{Results and Discussion}

It has been reported that amino acids such as tryptophan have strong electron-donating properties, ${ }^{21}$ and $p$-benzoquinone (BQ), which is hydrophobic in nature, is a strong electron acceptor. ${ }^{22}$ Herein, we have utilized this property of $\mathrm{BQ}$, which enters to
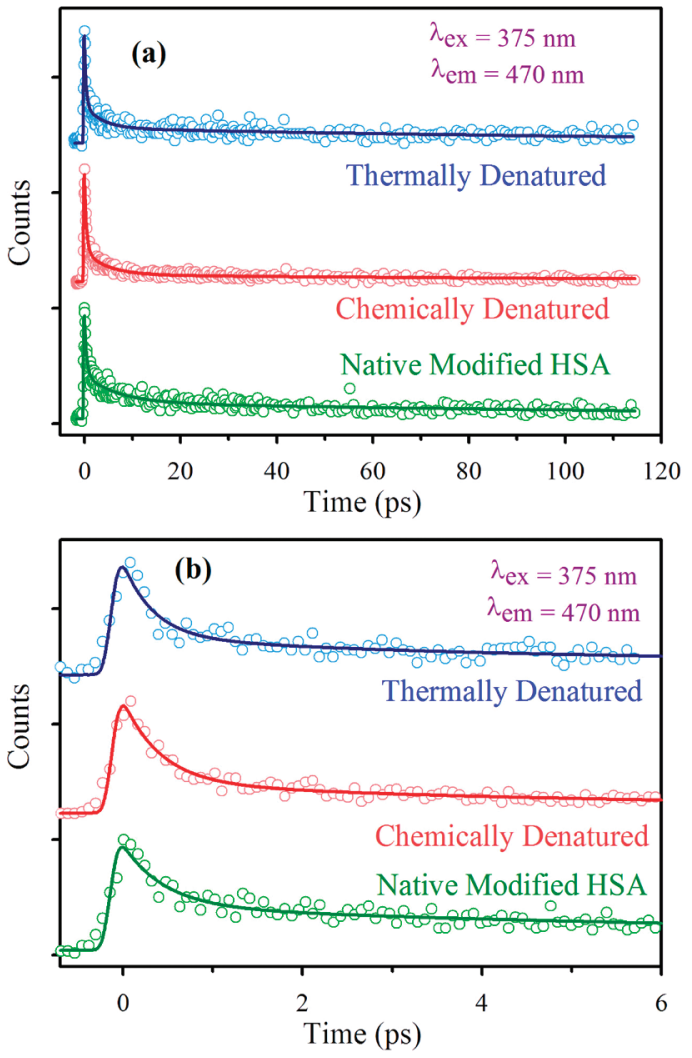

Figure 4. (a) Femtosecond time-resolved fluorescence transients of modified HSA in native and chemically and thermally denatured forms. The protein is excited at $375 \mathrm{~nm}$, and the transients were collected at $470 \mathrm{~nm}$. (b) Early dynamics at the same wavelengths.

the hydrophobic cavity of the protein and oxidized the tryptophan (Trp214) to kynurenine (Kyn214) as shown in Scheme 1. It can be seen from Figure 1a that a new absorption band at $353 \mathrm{~nm}$ with relatively broad bandwidth appears in the protein solution after the addition of ethanolic BQ. Although weak as compared to the $280 \mathrm{~nm}$ protein peak, the absorption band at $353 \mathrm{~nm}$ indicates the formation of Kyn214 in the model protein. ${ }^{23}$ The newly generated fluorescence probe with excitation and emission maxima at 350 and $450 \mathrm{~nm}$, respectively, is clearly observed from modified protein solution (inset of Figure 1a). The peak $(353 \mathrm{~nm})$ and the bandwidth of the excitation spectrum are identical to the modified protein absorption band in Figure 1a. Consistency of the excitation spectrum with that of the absorption and the invariance of the emission spectra with the excitation wavelength confirm the presence of single emissive species, i.e., Kyn214. The evolutions of the emission spectrum at the various levels of modifications are shown in Figure 1b. Initially, no fluorescence (except Trp214 emission at $350 \mathrm{~nm}$, excitation at $299 \mathrm{~nm}$ ) is observed from protein solutions, but modification is clearly demonstrated by the emission spectrum of the protein solution with increasing the concentration of BQ. Creation of an ultrafast component, i.e., 290 ps (18\%) (inset of Figure 1b) is the indication of newly generated fluorophore. The fluorescence decay transient of the modified protein solution (Figure 1c) shows three lifetime components: 160 ps (56\%), 1240 ps (30\%), and 4520 ps (15\%) with an average lifetime of 1130 ps. The anisotropy decay reveals a long component of $\sim 50 \mathrm{~ns}$, consistent with the hydrodynamic rotational relaxation time of the protein ${ }^{24}$ (inset of Figure 1c) in the solution.

Structural study on the protein is thus of interest, because modification may perturb the native structure and changes in 

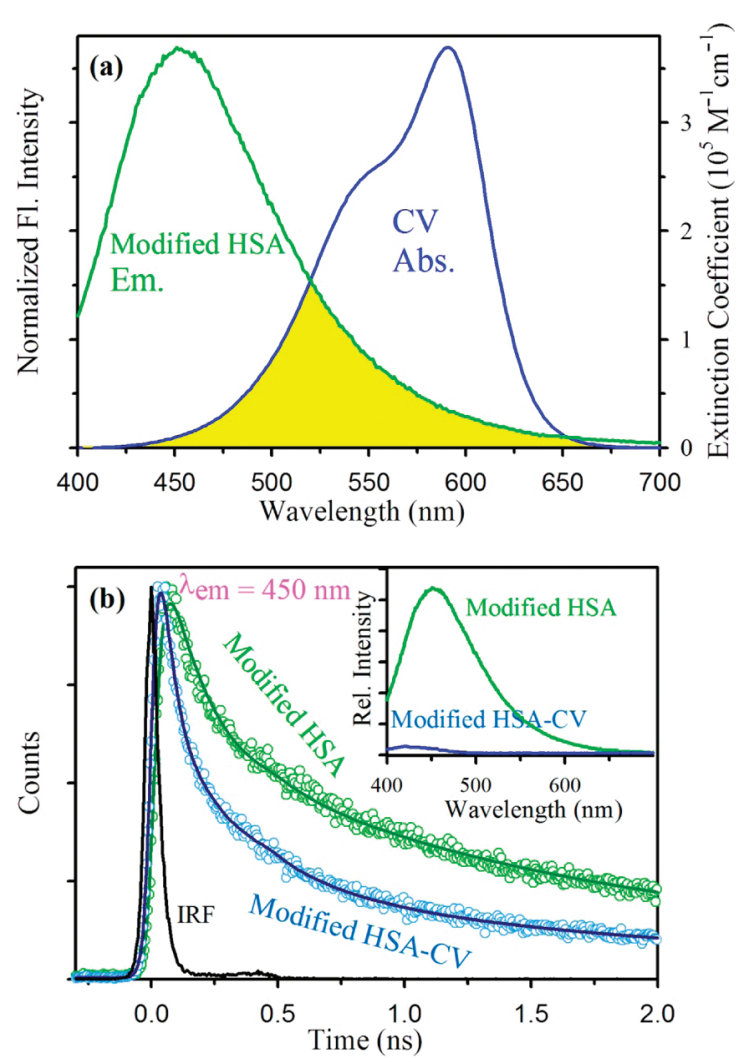

Figure 5. (a) Steady-state absorption spectra of CV (blue) and emission spectra of modified HSA (green). An overlapping zone between emission of modified HSA and absorption of acceptor CV is indicated as a yellow-shaded zone. (b) Picosecond-resolved fluorescence transients of modified HSA in the absence (green) and in the presence of acceptor $\mathrm{CV}$ (blue) (excitation at $375 \mathrm{~nm}$ ) collected at $450 \mathrm{~nm}$. Inset of panel b shows the steady-state emission spectrum of modified HSA in the presence (blue) and absence (green) of CV.

its native conformation could be translated into a loss of its biological function. Persistence of the structural integrity of the protein upon modification is confirmed by dynamic light scattering (DLS), near and far circular dichroism (CD) spectroscopy. The hydrodynamic diameter $\left(d_{\mathrm{H}}\right)$ of the modified protein (Figure 2a) as revealed from the DLS studies is consistent with that of its unmodified version. ${ }^{24}$ The temperaturedependent change of the $d_{\mathrm{H}}$ revealed the melting of the overall globular structure of the protein. The invariance of the thermal stability of the modified protein, compared that of the native one, is further confirmed by near-UV CD studies ${ }^{25}$ as shown in Figure $2 b$. From Figure $2 b$ it is evident that the CD signal at $268 \mathrm{~nm}$ indicative of the globular tertiary structure of the protein and the melting characteristics of the protein is consistent with that of the unmodified protein. From the DLS and near-UV CD studies it is also evident that the thermal unfolding above the melting temperature $\left(70{ }^{\circ} \mathrm{C}\right)$ is irreversible, analogous to the thermal denaturation property of the native protein. The effect of thermal denaturation on the secondary structure (far-UV CD) of the modified and unmodified protein is shown in Figure 3. From Figure 3a,b it is evident that the temperature-induced evolution of the far-UV CD spectra of the protein reveals a decrease in the overall ellipticity and the presence of an isodichroic point at $202 \mathrm{~nm}$ indicative of a cooperative loss of the $\alpha$-helix structure of the modified and unmodified proteins in a two-state-type transition. ${ }^{25}$ Persistence of the structural integrity of the protein upon modification is further confirmed by monitoring ellipticity at $222 \mathrm{~nm}$ (far-UV CD). Both the proteins exhibit comparable sigmoidal transition indicating the
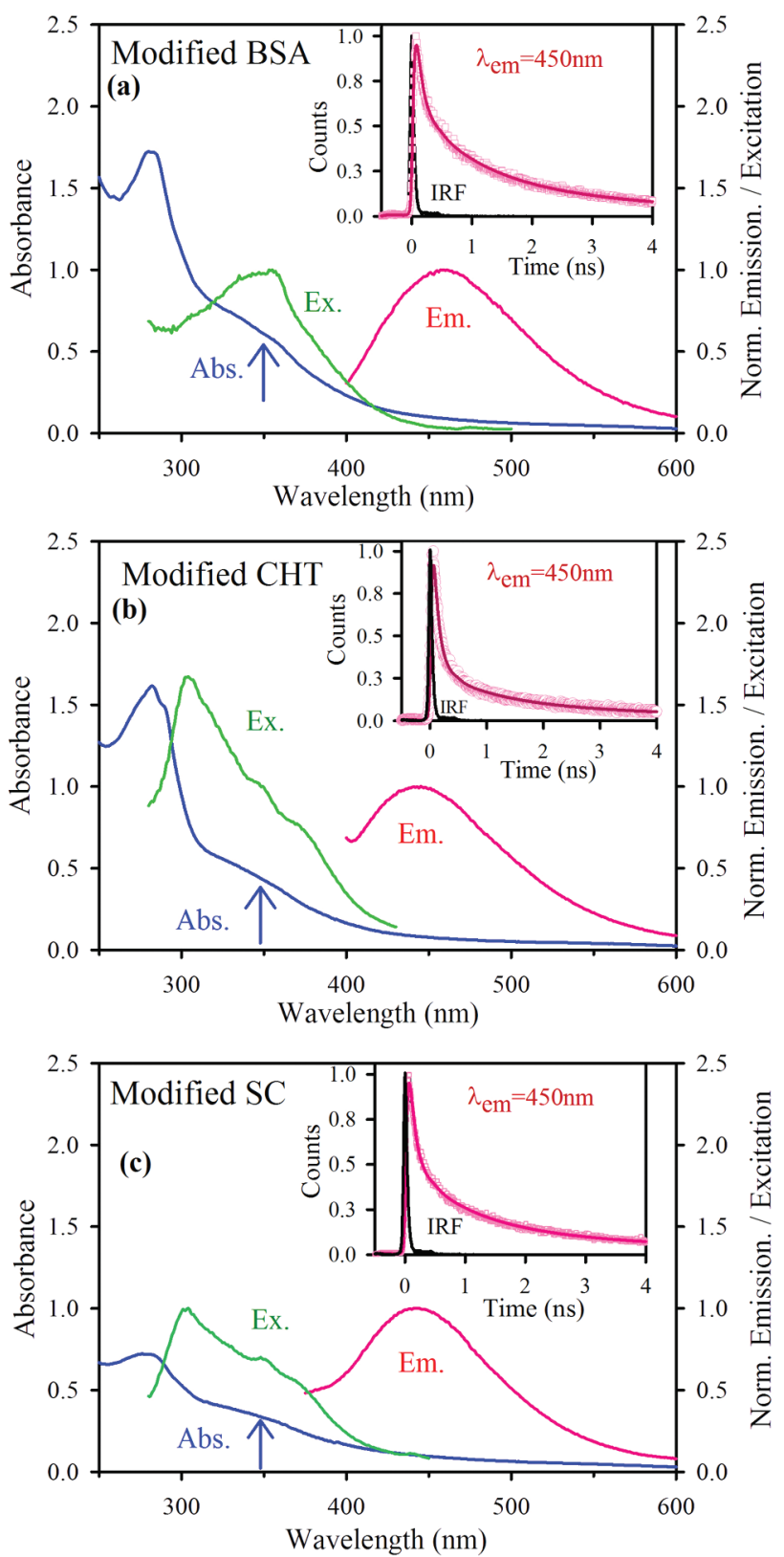

Figure 6. Absorption (a), excitation (b), and emission (c) spectra of modified bovine serum albumin (BSA), modified $\alpha$-chymotrypsin (CHT), and modified subtilisin Carlsberg (SC). Insets show their picosecond-resolved fluorescence transients.

similar unfolding pathways ${ }^{25,26}$ revealing melting temperature $\left(T_{\mathrm{m}}\right)$ to be $70{ }^{\circ} \mathrm{C}$. The observation is consistent with DLS and near-UV CD studies (Figure 2).

The efficacy of the probe Kyn214 for the exploration of dynamical information of the protein within its structural integrity as confirmed by $\mathrm{CD}$ studies is now a point to be discussed. We have compared the efficacy of the Kyn214 to report the dynamics of solvent molecules in the protein cavity.

TABLE 1: Multiexponential Fitting Parameters of the Picosecond-Resolved Fluorescence Transients of Various Proteins

\begin{tabular}{lcclc}
\hline \multicolumn{1}{c}{ protein } & $\tau_{1}(\mathrm{~ns})$ & $\tau_{2}(\mathrm{~ns})$ & \multicolumn{1}{c}{$\tau_{3}(\mathrm{~ns})$} & $\tau_{\mathrm{av}}(\mathrm{ns})$ \\
\hline modified-BSA & $0.110(59 \%)$ & $1.006(28 \%)$ & $3.892(13 \%)$ & 0.852 \\
modified-CHT & $0.091(82 \%)$ & $1.045(12 \%)$ & $5.112(6 \%)$ & 0.506 \\
modified-SC & $0.102(68 \%)$ & $0.941(22 \%)$ & $4.273(10 \%)$ & 0.703
\end{tabular}



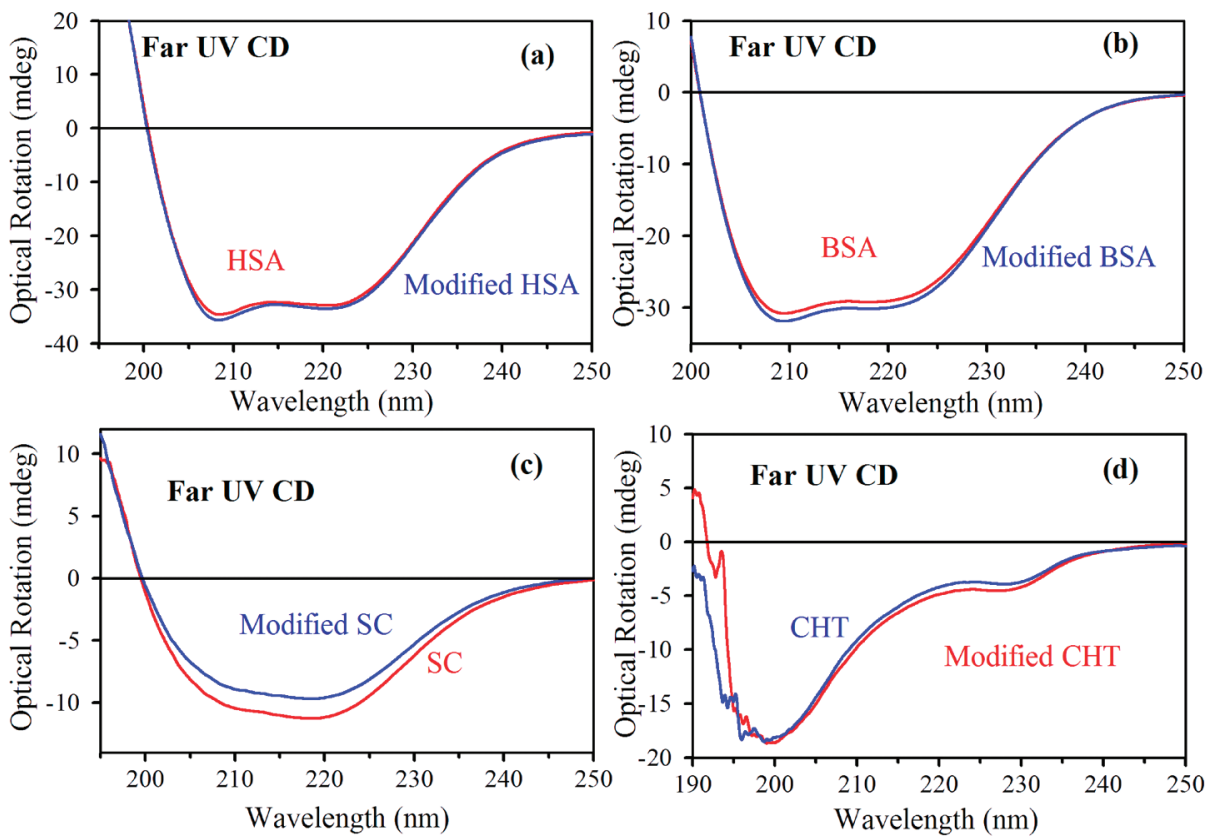

Figure 7. Far-UV CD spectrum of human serum albumin (HSA) (a), bovine serum albumin (BSA) (b), subtilisin Carlsberg (SC) (c), and $\alpha$-chymotrypsin (CHT) (d) before and after modification. Here each protein and its modified form have the same concentration.

By using femtosecond-resolved fluorescence studies, the dynamical time constants of the solvent molecules in the protein cavity have been explored to be ultrafast and multiexponential. ${ }^{27,28}$ It has also been demonstrated that the chemical denaturation of the protein allows more bulk type water in the protein cavity, revealing faster time constants in the solvation dynamics. ${ }^{27,28}$ A systematic study on the spectroscopy and dynamics of kynurenine in different solvents has been presented by Vauthey et al. ${ }^{16}$ The usefulness of the probe (kynurenine) as a solvation reporter has also been established. ${ }^{16}$ The main excited-state deactivation channel of the probe depends on the interaction of the solvent molecules through hydrogen-bonding ability. However, it has to be noted that reorganization of hydrogen-bondforming solvents with respect to the probe solute is extremely important for the formation of solute-solvent hydrogen bond formation. ${ }^{29}$ The rate of reorganization of the water molecules around the solute probe (here kynurenine) is limited by the dynamics of solvation. Thus, the two dynamical time scales in a system should be intimately related. The femtosecond fluorescence studies on the probe in various bulk solvents demonstrate that the excited-state lifetime of the probe in water (0.9 ps) significantly increases (more than $1 \mathrm{~ns}$ ) in DMSO, where the probe-solvent hydrogen-bonding interaction is absent. Herein, the femtosecond-resolved time constants of the fluorescence transient of the Kyn214 in the native protein (Figure 4) are $0.46 \mathrm{ps}(65 \%), 6.8 \mathrm{ps}(22 \%)$, and $157 \mathrm{ps}(13 \%)$, revealing the interaction of the probe with water molecules in the cavity. The time constants 0.34 ps $(73 \%), 3.7$ ps (17\%), and 128 ps $(10 \%)$ at $75{ }^{\circ} \mathrm{C}$, and $0.42 \mathrm{ps}(78 \%), 4.7 \mathrm{ps}(18 \%)$, and $128 \mathrm{ps}$ (4\%) at $\mathrm{pH}=12$ in the denatured states distinctly reveal more water accessibility of the probe Kyn214 compared to that in native conformation consistent with the previous results. ${ }^{27,28}$ Recently, Vauthey et al. ${ }^{30}$ have explored the photophysics and photochemistry of the kynurenine covalently attached to amino acids and to a model protein lysozyme. It has been demonstrated that the increase of photoactivity of the kynurenine covalently attached to amino acids and protein is due to the decrease of the rate of solvent-assisted internal conversion.

In order to establish more general use of the modified probe Kyn214, we have deliberate dipolar interaction of the probe with a surface-bound organic dye, crystal violet (CV). ${ }^{31}$ From Figure 5a, a significant spectral overlap of the Kyn214 emission with the absorption spectrum of $\mathrm{CV}$ is evident. The significant quenching in the steady-state emission and picosecond-resolved fluorescence transient (at $450 \mathrm{~nm}$ ) as a consequence of the donor-acceptor dipolar interaction is clearly evident from Figure 5b. From this, we have estimated a Förster resonance energy transfer (FRET) efficiency to be $73 \%$ and the characteristic distance, $R_{0}$ of the FRET pair is $2.72 \mathrm{~nm}$. FRET from Kyn214 (donor) in the native protein to a well-known surfacebound organic dye $\mathrm{CV}$ is successfully demonstrated and donor-acceptor distance of $2.29 \mathrm{~nm}$ is found to be consistent with that of the estimated value $(1.84 \mathrm{~nm})$ from X-ray crystallographic structure of the protein. ${ }^{32,33}$

In order to establish the generality of this electron-transfer mechanism, we have performed similar experiments on a number of proteins and found that $\mathrm{BQ}$ is able to oxidize the Trp residues in any kind of protein. Figure $6, \mathrm{a}, \mathrm{b}$ and $\mathrm{c}$, describes the absorption, excitation, and emission spectra of modified bovine serum albumin (BSA), modified $\alpha$-chymotrypsin (CHT), and modified subtilisin Carlsberg (SC), respectively. The numbers of tryptophan residues in the proteins are two, seven, and one, respectively. While BSA and CHT contain buried tryptophan residues, the protein SC contains waterexposed single tryptophan residue. As evident from Figure 6, like HSA, all the proteins contain a broad absorption spectra peaking at $350 \mathrm{~nm}$ which indicates the formation of kynurenine. The excitation and emission spectra are also clear indications of the formation of newly generated fluoroprobe, kynurenine. Insets of Figure 6 show the picosecond-resolved multiexponential (shown in Table 1) fluorescence transients of modified BSA, modified CHT, and modified SC.

Persistence of the structural integrity of the proteins upon modification is confirmed by far-UV CD experiment. Figure 7 indicates that there is insignificant loss of overall ellipticity upon modification of HSA, BSA, SC, and CHT proteins which indicates that BQ is good to oxidize Trp of any kind of proteins within structural integrity. Again, when the ellipticity was monitored at $222 \mathrm{~nm}$ (for BSA and SC) and at $232 \mathrm{~nm}$ (for $\mathrm{CHT}$ ), the thermal denaturation of the modified proteins exhibits 

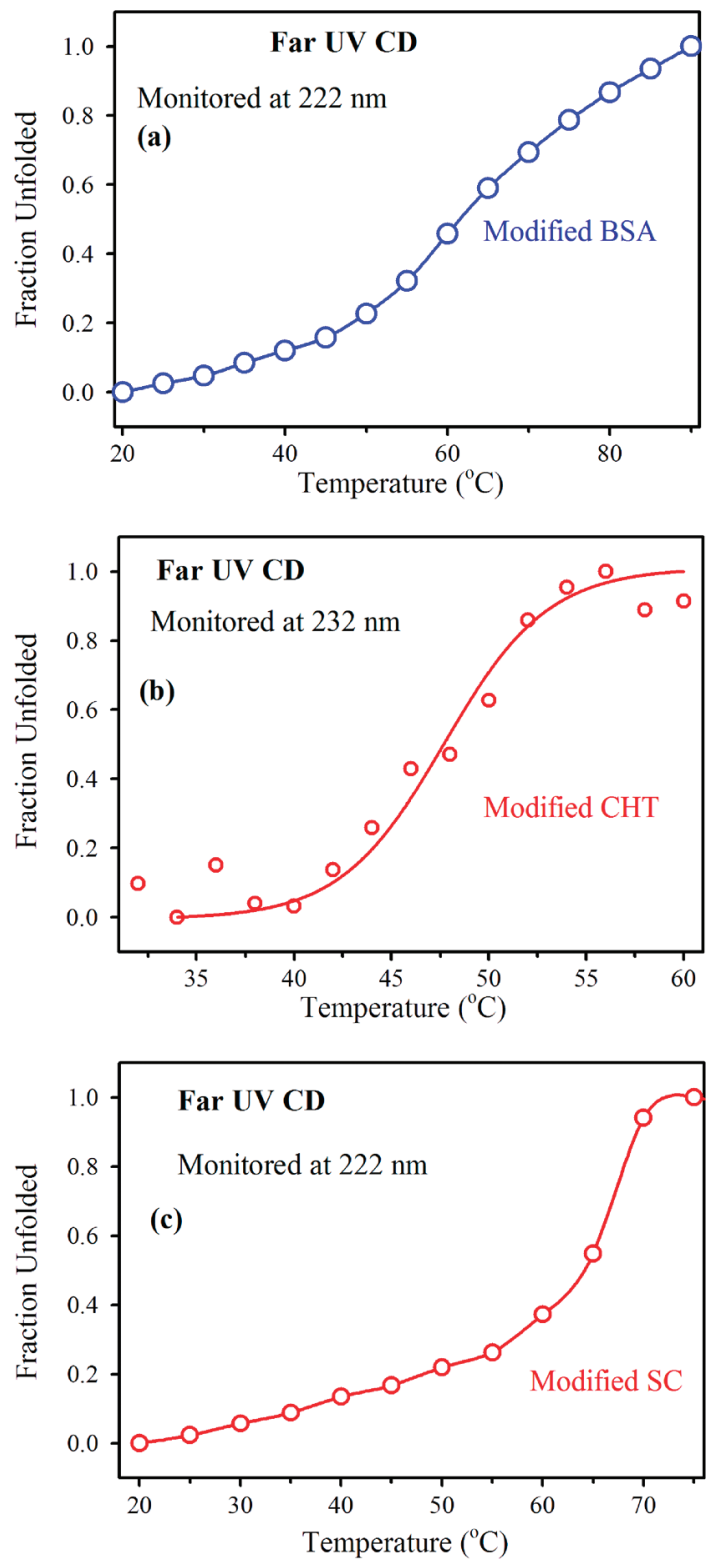

Figure 8. Temperature dependence of the far-UV CD spectrum of the modified bovine serum albumin (BSA) (a), modified $\alpha$-chymotrypsin (CHT) (b), and modified subtilisin Carlsberg (SC) (c) in phosphate buffer $(\mathrm{pH} 7.0,20 \mathrm{mM})$. Transition temperatures $\left(T_{\mathrm{m}}\right.$, the midpoint of unfolding transition) are $\sim 70, \sim 50$, and $\sim 62{ }^{\circ} \mathrm{C}$, respectively, which are consistent with those of the native proteins. The solid lines represent guides to eyes.

a cooperative sigmoidal behavior with transition temperature $\left(T_{\mathrm{m}}\right)$ similar to those of the unmodified ones ${ }^{26}$ (shown in Figure 8). Our observations indicate that unfolding pathways are not perturbed upon modification with ethanolic BQ.

Figure 9a shows the rate of formation of AMC product upon enzymatic activity of CHT and modified CHT on AAF-AMC in phosphate buffer. Figure $9 \mathrm{~b}$ shows time-dependent product concentration as a result of enzymatic activity of SC and modified SC on CBZ-GGL-pNA in phosphate buffer. Table 2 lists the specific activity (in units/mg) of CHT, modified CHT, SC, and modified SC. From Table 2, we find that the specific activities of the CHT and SC are close to those of modified CHT and modified SC, respectively, which depicts that oxidation of Trp has minimal effect on the biological function of the protein.
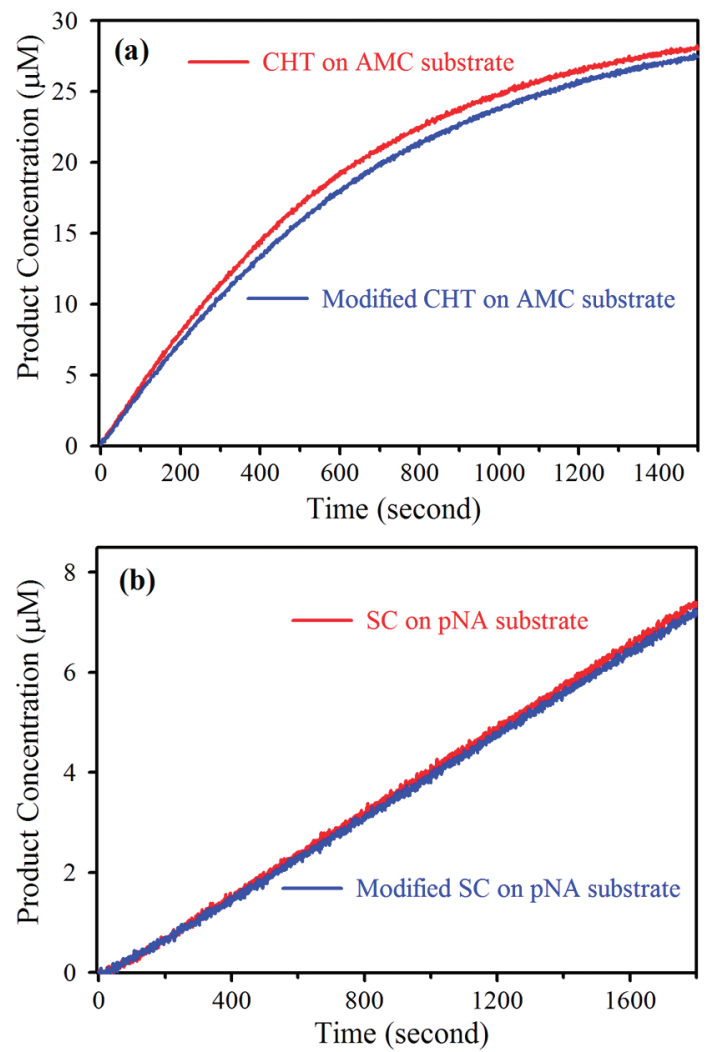

Figure 9. (a) Concentrations of the product as a function of time. Concentrations of CHT and AAF-AMC were 1 and $35 \mu \mathrm{M}$, respectively. (b) Concentrations of the product as a function of time. Concentrations of SC and CBZ-GGL-pNA were 1 and $185 \mu \mathrm{M}$, respectively.

TABLE 2: Comparison of Specific Activity in Units/mg of CHT ${ }^{a}$ Modified CHT, SC, ${ }^{b}$ and Modified SC

\begin{tabular}{lc}
\hline \multicolumn{1}{c}{ system } & specific activity (units/mg) \\
\hline CHT & 22.89 \\
modified CHT & 22.41 \\
SC & 4.41 \\
modified SC & 4.38
\end{tabular}

${ }^{a}$ One unit of CHT will hydrolyze $1.0 \mu \mathrm{mol}$ of AAF-AMC per minute at $\mathrm{pH} 7.0$ at $25{ }^{\circ} \mathrm{C} .{ }^{b}$ One unit of SC will hydrolyze 1.0 $\mu \mathrm{mol}$ of CBZ-GGL-pNA per minute at $\mathrm{pH} 7.0$ at $25^{\circ} \mathrm{C}$.

\section{Conclusion}

In conclusion, an efficient intrinsic fluorescence probe, i.e., Kyn214, has been successfully modified from Trp214 in a model protein cavity through an electron-transfer reaction. It has to be noted that we have studied a number of proteins and found that BQ is good to oxidize Trp in any proteins. Dynamics of hydration in the protein cavity as revealed by Kyn214 is also demonstrated by femtosecond studies and found to be consistent with other studies. Practical applications of protein-bound kynurenine as an energy transfer probe is also established here. Our study further demonstrates that oxidation of tryptophan does not affects the biological functionality of the protein. With simple modification, such intrinsic fluorophore is likely to find use in spectroscopic studies of protein, thereby making these studies much more widely accessible. The intense emission and the excitation wavelength at near-visible wavelength $(\sim 375 \mathrm{~nm})$ and eligibility for the spectroscopic studies of a protein within structural integrity make it an attractive fluoroprobe as an 
alternative to tryptophan. Our studies would find out the relevance in the spectroscopic investigation of the protein molecule.

Acknowledgment. N.G. and A.M. thank CSIR, India, for the fellowships. We thank DST, India, for a financial grant (SR/ SO/BB-15/2007).

\section{References and Notes}

(1) Lakowicz, J. R. In Principles of Fluorescence Spectroscopy, 2nd ed.; Kluwer Academic/Plenum Publishers: New York, 1999.

(2) Beechem, J. M.; Brand, L. Annu. Rev. Biochem. 1985, 54, 43.

(3) Mannuzzu, L. M.; Moronne, M. M.; Isacoff, E. Y. Science 1996, $271,213$.

(4) Cohen, B. E.; McAnaney, M. T.; Park, E. S.; Jan, Y. N.; Boxer, S. G.; Jan, L. Y. Science 2002, 296, 1700.

(5) Shi, L.; Paoli, V. D.; Rosenzweig, N.; Rosenzweig, Z. J. Am. Chem. Soc. 2006, 128, 10378.

(6) Royer, A. C. Chem. Rev. 2006, 106, 1769.

(7) Shen, X.; Knutson, J. R. J. Phys. Chem. B 2001, 105, 6260.

(8) Zhong, D.; Pal, S. K.; Zhang, D.; Chan, S. I.; Zewail, A. H. Proc. Natl. Acad. Sci. U.S.A. 2002, 99, 13.

(9) Zhang, H.; Andrekopoulos, C.; Joseph, J.; Chandran, K.; Karoui, H.; Crow, J. P.; Kalyanaraman, B. J. Biol. Chem. 2003, 278, 24078.

(10) Anderson, L. B.; Maderia, M.; Ouellette, A. J.; Putnam-Evans, C.; Higgins, L.; Krick, T.; MacCoss, M. J.; Lim, H.; Yates, J. R.; Barry, B. A. Proc. Natl. Acad. Sci. U.S.A. 2002, 99, 14676.

(11) Vazquez, S.; Aquilina, J. A.; Jamie, J. F.; Sheil, M. M.; Truscott, R. J. J. Biol. Chem. 2002, 277, 4867. 806.

(12) Cleveland, D. W.; Rothstein, J. D. Nat. Rev. Neurosci. 2001, 2,

(13) Aquilina, J. A.; Carver, J. A.; Truscott, R. J. Biochemistry 2000, 39,16176 .
(14) Aquilina, J. A.; Carver, J. A.; Truscott, R. J. Biochemistry 1999, 38,11455 .

(15) Stachel, S. J.; Habeeb, R. L.; Van Vranken, D. A. J. Am. Chem. Soc. 1996, 118, 1225.

(16) Sherin, S. P.; Grilj, J.; Tsentalovich, P. Y.; Vauthey, E. J. Phys. Chem. B 2009, 113, 4953.

(17) Abou-Zied, O. K.; Al-Shihi, O. I. K. J. Am. Chem. Soc. 2008, 130, 10793.

(18) Sarkar, R.; Narayanan, S. S.; Pålsson, L.; Dias, F.; Monkman, A.; Pal, S. K. J. Phys. Chem. B 2007, 111, 12294.

(19) Wu, P.; Brand, L. Anal. Biochem. 1994, $218,1$.

(20) Chen, J.; Flaugh, S. L.; Callis, P. R.; King, J. Biochemistry 2006, 45,11552 .

(21) Si, S.; Mandal, T. K. Chem.-Eur. J. 2007, 13, 3160

(22) Burda, C.; Green, T. C.; Link, S.; El-Sayed, M. A. J. Phys. Chem. $B$ 1999, 103, 1783.

(23) Yamashita, S.; Nishimoto, E.; Szabo, A. G.; Yamasaki, N. Biochemistry 1996, 35, 531.

(24) Mitra, R. K.; Sinha, S. S.; Pal, S. K. Langmuir 2007, 23, 10224.

(25) Balsera, M.; Menéndez, M.; Sáiz, J. L.; Rivas, J. D. L.; Andreu, J. M.; Arellano, J. B. Biochemistry 2004, 43, 14171.

(26) Kamal, J. K. A.; Xia, T.; Pal, S. K.; Zhao, L.; Zewail, A. H. Chem. Phys. Lett. 2004, 387, 209.

(27) Qiu, W.; Zhang, L.; Okobiah, O.; Yang, Y.; Wang, L.; Zhong, D.; Zewail, A. H. J. Phys. Chem. B 2006, 110, 10540.

(28) Kamal, J. K. A.; Zhao, L.; Zewail, A. H. Proc. Natl. Acad. Sci. U.S.A. 2004, 101, 13411.

(29) Chakraborty, S.; Bandyopadhyay, S. J. Phys. Chem. B 2007, 111, 7626.

(30) Sherin, P. S.; Grilj, J.; Kopylova, L. V.; Yanshole, V. V.; Tsentalovich, Y. P.; Vauthey, E. J. Phys. Chem. B 2010, 114, 11909.

(31) Santhanalakshmi, J.; Balaji, S. Colloids Surf., A 2001, 186, 173.

(32) Wardell, M.; Wang, Z.; Ho, J. X.; Robert, J.; Ruker, F.; Ruble, J.;

Carter, D. C. Biochem. Biophys. Res. Commun. 2002, 291, 813.

(33) Zunszain, P. A.; Ghuman, J.; Komatsu, T.; Tsuchida, E.; Curry, S. BMC Struct. Biol. 2003, 3, 6 .

JP105943D 